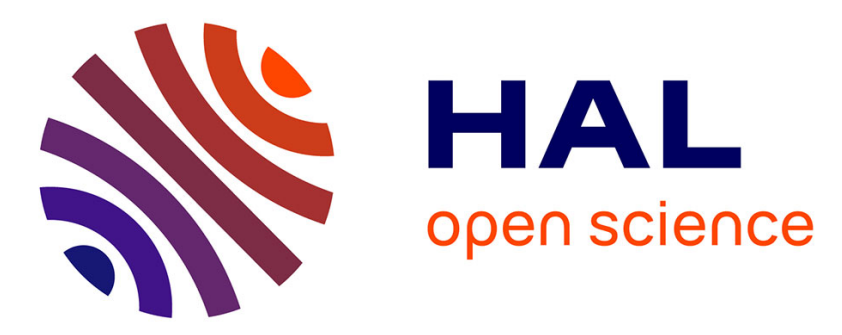

\title{
Un discours féministe? Les représentations de lectrices dans les romans pédagogiques de Louise d'Epinay, Caroline de Genlis et Isabelle de Charrière
} Sandrine Aragon

\section{- To cite this version:}

Sandrine Aragon. Un discours féministe? Les représentations de lectrices dans les romans pédagogiques de Louise d'Epinay, Caroline de Genlis et Isabelle de Charrière. Women in French Studies, 2002, Tenth Anniversary volume, pp. 144-152. hal-02461591

\section{HAL Id: hal-02461591 \\ https://hal.science/hal-02461591}

Submitted on 4 Feb 2020

HAL is a multi-disciplinary open access archive for the deposit and dissemination of scientific research documents, whether they are published or not. The documents may come from teaching and research institutions in France or abroad, or from public or private research centers.
L'archive ouverte pluridisciplinaire HAL, est destinée au dépôt et à la diffusion de documents scientifiques de niveau recherche, publiés ou non, émanant des établissements d'enseignement et de recherche français ou étrangers, des laboratoires publics ou privés. 


\section{SANDRINE ARAGON}

\section{Un discours féministe? Les représentations de lectrices dans les romans pédagogiques de Louise d'Epinay, Caroline de Genlis et Isabelle de Charrière}

- "Jamais fille chaste n'a lu de romans..."1 (Préface de La Nouvelle Héloïse)

- "Une femme bel esprit est le fléau de son mari, de ses enfants, de ses amis, de ses valets, de tout le monde." (Rousseau, Emile ou de l'éducation)

- "Toute fille lettrée restera fille toute sa vie tant qu'il n'y aura que des hommes sensés sur la terre" ${ }^{3}$ (Livre cinquième de Emile ou de l'éducation).

Les discours de Rousseau sur l'éducation ont profondément marqué les lecteurs de la fin du XVIIIe siècle. Parmi ses nombreuses lectrices, certaines n'ont toutefois pas hésité à s'engager dans la voie de l'écriture pédagogique, malgré les anathèmes du grand homme sur les "femmes auteurs". La rédaction d'ouvrages pédagogiques semble encore le seul type d'écriture qu'une femme de la bonne société puisse justifier à l'époque, elle peut arguer qu'il s'agit d'une démarche utilitaire et dans la droite ligne de ses fonctions maternelles et donc éviter les accusations de bel esprit frivole.

Face aux besoins de l'éducation féminine et à la demande de formation, des femmes cultivées de la haute société ont suivi le chemin tracé par Mme de Maintenon un siècle plus tôt et par les écrits de Mme de Lambert. Mme d'Epinay, avec le premier volume des Conversations d'Emilie (1774) a dessiné les principes d'une éducation éclairée fondée sur la discussion entre mère et fille ; Mme de Genlis, après avoir écrit des pièces de théâtre pour l'instruction des jeunes filles, rédige une somme pédagogique avec son roman Adèle et Théodore en 1782.

Ces deux femmes auteurs connaissent parfaitement les théories de Rousseau, elles l'ont bien lu et elles l'ont toutes deux fréquenté : quels discours tiennent-elles sur l'éducation des filles ? Comment se situent-elles par rapport à ce discours masculin dominant ?

\section{Des discours féminins qui prennent position par rapport aux discours masculins contemporains sur l'éducation des filles}

Mme d'Epinay et Mme de Genlis ont toutes deux écrit des fictions nourries de principes pédagogiques; leurs discours diffèrent toutefois radicalement de ceux des hommes contemporains, auteurs de fictions, qui s'intéressent à l'éducation féminine.

Après Rousseau, peu d'hommes se sont illustrés à la fin du XVIIIe siècle par leurs théories de l'éducation. On retrouve toutefois la question de l'éducation féminine dans les fictions écrites par des hommes, notamment dans les romans libertins.

Le célèbre roman de Choderlos de Laclos, Les Liaisons dangereuses, est paru en 1782, soit la même année que Adèle et Théodore. En 1784, Rétif de la Bretonne s'est également intéressé au problème dans La Paysanne perverti,. Mirabeau,a proposé aussi un récit d'éducation de jeune fille dans le roman Le rideau levé ou l'éducation de Laure à la même époque. Le topos de la jeune fille innocente pervertie par un libertin (qui utilise des livres pour la séduire) très présent au début du siècle, est réutilisé dans ces romans. L'éducation se fait toujours au détriment de la vertu, que ce soit pour la jeune Cécile de Volanges ou pour la marquise de Merteuil, autodidacte très instruite MAIS libertine. La collusion entre lecture et sexualité

\footnotetext{
${ }^{1}$ Rousseau, Préface de Julie ou la Nouvelle Héloïse (édition René Pomeau, Paris, Garnier, 1960, p. 4).

${ }^{2}$ Rousseau, Emile ou de l'éducation, livre cinquième (édition Michel Launay, Paris, Garnier-Flammarion, 1966, p. 536).

${ }^{3}$ Rousseau, Emile ou de l'éducation, livre cinquième (Op. Cit., p. 537).
} 
débauchée, est constamment mise en évidence dans ces fictions, pour le plus grand plaisir des lecteurs libertins ${ }^{4}$.

Face à ces images de jeunes filles perverties par la lecture, Mme d'Epinay, Mme de Genlis et Mme de Charrière proposent des personnages de jeunes lectrices extrêmement sages, qui s'instruisent et acquièrent leur sagesse par la lecture. Le discours de ces femmes rompt donc totalement avec le topos de la lecture féminine libertine présent dans bon nombre de fictions du XVIIIe siècle ; par bien des points il se rapproche des préceptes préconisés par Rousseau pour éduquer des filles vertueuses et chastes.

Elles remplacent le couple "lectrice et libertin séducteur" par celui de la "mère veillant sur sa fille". Ces auteurs, lectrices de Rousseau, retiennent dans leurs principes éducatifs des leçons du grand homme : elles décrivent toutes trois des jeunes filles guidées par des mères très présentes ; elles ne sont point mises en nourrice ou au couvent mais élevées, de leur plus jeune âge jusqu'à leur mariage, par une mère préceptrice fidèle, à l'instar de Sophie dans Emile ou de l'éducation.

Toutes ces mères préconisent une éducation au grand air et laissent leurs filles grandir et se développer librement sans coquettes parures, comme le conseille vivement Rousseau. La mère des Conversations d'Emilie invite la fillette à aller courir dans le parc plutôt que de rester dans sa chambre. Adèle et Théodore de Mme de Genlis, s'ouvre sur une lettre de la baronne d'Almane annonçant son départ de Paris avec son mari et ses deux enfants en bas âge pour le Languedoc ; ils s'éloignent pour éduquer leurs enfants à la campagne.

Toutes deux insistent également sur le fait qu'elles ne veulent surtout pas faire de leurs filles des savantes mais des jeunes femmes sages et vertueuses qui deviendront des épouses fidèles et des mères attentives. La baronne d'Almane écrit même dans l'œuvre de Mme de Genlis :

"... on doit éviter avec soin d'enflammer l'imagination des femmes et d'exalter leurs têtes ; elles sont nées pour une vie monotone et dépendante." 5

Par de tels discours, elles se défendent ainsi contre toutes les attaques à l'encontre des femmes savantes pédantes et distinguent leurs filles du "bel esprit qui restera fille toute sa vie" selon Rousseau.

Cependant, ces femmes auteurs dans leurs discours ne suivent pas totalement celui du grand homme, elles s'en écartent notamment sur la qualité et la quantité des enseignements donnés aux jeunes héroïnes.

Elles ne remettent pas au mari le soin d'instruire leurs femmes à leur convenance comme le souhaite Rousseau dans l'Emile. Elles jugent l'éducation essentielle pour qu'une femme soit heureuse dans son foyer ${ }^{6}$ et défendent le bonheur d'être instruite. Mme d'Epinay rappelle qu'elles doivent éduquer leurs enfants et que "pour instruire, il faut être instruite"7. Son

\footnotetext{
${ }^{4}$ Même si Choderlos de Laclos s'écarte des anathèmes de Rousseau. Son essai sur l'éducation féminine, publié un an après Les Liaisons dangereuses, s'inspire du maître mais arrive à d'autres conclusions. Voir Choderlos de Laclos, De l'éducation des femmes (1783), (Grenoble, Jérôme Millon, 1991).

${ }^{5}$ Mme de Genlis, Adèle et Théodore (Paris, Michel Lambert, 1782, vol. 1, lettre X, p. 39).

6 "Rousseau peut bien enfermer les femmes dans leur foyer, Mme d'Epinay leur donne la grande recette pour s'en évader. Elle renverse le principe de dépendance établi par Jean-Jacques. C'est là la critique la plus évidente jamais faite en ce temps à la doctrine de Rousseau. C'est également l'incomparable grandeur de Mme d'Epinay d'avoir compris deux siècles avant beaucoup d'autres que les femmes devraient œuvrer elles-mêmes pour leur bonheur et ne pas s'en remettre uniquement aux hommes" selon Elisabeth Badinter (Emile, Emilie ou l'ambition féminine au XVIIIe siècle, Paris, Flammarion, 1983, p. 395).

${ }^{7}$ Mme d'Epinay, Les Conversations d'Emilie, Douzième conversation (Oxford, Voltaire Foundation, 1996, p. 238).
} 
héroïne se plaint de son manque d'instruction et sa fille Emilie, qui n'a pas dix ans dans le premier volume des Conversations, lit, réalise des synthèses, des notes de lecture et commente avec sa mère des auteurs tel que Plutarque. Quant à Mme de Genlis, elle multiplie les activités et les enseignements d'Adèle dans son roman : une nourrice anglaise lui enseigne l'anglais à six mois, afin qu'elle le parle comme sa langue maternelle ; un maître de dessin italien l'entraîne très jeune en lui parlant italien. Elle parle et lit ensuite en trois langues, dessine, joue de la musique et est capable de s'occuper, d'apprendre et d'enseigner dans de multiples domaines (histoire, géographie, littérature, géométrie...) sans oublier bien sûr la morale et la religion qui occupent une grande place dans son éducation.

Les images de lectrices présentes dans ces romans pédagogiques diffèrent donc totalement des images de "jeunes filles perverties par leurs lectures". De vastes domaines de connaissances sont ouverts aux jeunes filles dans les romans des auteurs féminins, sans péril. L'objectif déclaré est de former de jeunes femmes autonomes, sachant s'orienter sur les chemins du savoir et apprendre en autodidacte tout en formant les autres. La différence est nette avec les ouvrages des auteurs masculins contemporains, qui au mieux déplorent dans les fictions l'éducation inadaptée des couvents ou les lectures féminines (trop savantes pour leur entendement ou trop romanesques pour qu'elles soient heureuses dans leur vie quotidienne). Les œuvres des femmes auteurs offrent de véritables programmes de formation à leurs lectrices virtuelles.

\section{Des programmes éducatifs pour aider les mères à former leurs filles}

Ce sont des romans féministes avant la lettre, destinés à faire avancer la condition féminine. Les auteurs ne se contentent pas de montrer que des jeunes filles peuvent lire sans danger et apprendre avec profit, Mme d'Epinay et Mme de Genlis incluent également dans leurs fictions des exercices et des programmes gradués en fonction de l'âge de leurs héroïnes. Mme d'Epinay, dans Les Conversations d'Emilie, explique comment accompagner les lectures de l'enfant : lui faire copier et résumer les passages difficiles pour son âge, lui montrer les incohérences de certains textes littéraires.

Mme de Genlis donne à la fin du roman le programme de lecture d'Adèle depuis l'âge de six ans jusqu'à vingt-deux ans. Elle décrit et explique certains exercices qui visent à développer l'esprit critique de ses deux enfants : la mère pédagogue a imaginé par exemple une correspondance fictive entre un frère et une sœur séparés ; lui est à Paris, elle en province. Il fait de mauvaises lectures et des liaisons dangereuses, il en parle à sa sœur dans ses lettres ; elle lui répond en argumentant pour lui montrer les dangers encourus et les erreurs de ses raisonnements. En donnant une à une les lettres de ce garçon imaginaire à sa fille, elle l'intéresse au personnage et lui confie la tâche de guider sur le droit chemin le jeune homme par son argumentation. Elles corrigent ensemble les lettres d'Adèle et la mère lui lit ensuite sa version de la lettre, elle procède donc ainsi à une évaluation avec correction de la rédaction de l'élève. De plus, elle met en valeur par cet exercice les défauts de certains livres et développe l'esprit critique de la jeune fille.

Même si son rôle de femme honnête consiste à obéir et à se taire, Adèle est formée pour être semblable à Julie, dirigeant son domaine, plutôt qu'à Sophie. Son éducation n'a rien à envier à celle de son frère. Elle bénéficie tout comme lui des sorties éducatives : visites d'usines, de manufactures accompagnées de lectures de l'Encyclopédie. Elle effectue avec ses parents le voyage initiatique en Europe réservé traditionnellement aux garçons. Pendant ces voyages, la formation ne cesse pas un instant et elle est mixte : les parents et les enfants rendent compte de leurs lectures au groupe puis les commentent et les critiquent ensemble.

Ces textes accordent donc aux femmes les mêmes capacités intellectuelles qu'aux hommes et ils expliquent aux lectrices réelles comment développer l'esprit des nouvelles générations de 
femmes. Bien plus, ils s'opposent aux allégations de Rousseau et de tous les contempteurs des femmes auteurs et mettent en valeur les femmes écrivains et leurs oeuvres

Mme d'Epinay loue les pièces de théâtres éducatives de Mme de Genlis, ses premières œuvres contemporaines des Conversations d'Emilie. Au sein d'un petit conte introduit dans la douzième conversation :

"Ces comédies charmantes étaient l'ouvrage d'une dame de la cour, célèbre par les grâces de son esprit et par la diversité de ses talens. Elle s'était soustraite à la société dont elle faisait le charme, pour consacrer son temps à un but infiniment plus intéressant et plus noble. Il était bien naturel qu'à la cour de Régentine il se trouvât une femme d'un mérite si rare; mais personne ne pouvait concevoir, comment une Princesse aussi éclairée ne lui avait pas confié l'éducation de ses filles."

Elle donne également les œuvres de Mme de Lambert à lire à Emilie, en parallèle avec la lecture des Méditations d'un auteur masculin, et elle lui prédit qu'elle verra très vite le texte qu'elle préfère :

"Mère : (...) je vous donnerai moi les cahiers d'une femme d'un grand mérite de ma connaissance.

Emilie : Qu'est-ce qu'il y a dans ces cahiers?

Mère : Elle a fait l'extrait des vies des hommes illustres de Plutarque, à l'usage d'une jeune personne qui en a singulièrement profité. Vous savez ce que c'est qu'un extrait, et vous comprenez qu'elle a rapproché les traits les plus remarquables de tous les grands personnages de l'antiquité. Si cela vous convient, après chaque Méditation vous lirez un de ces extraits; nous verrons lequel de ces deux ouvrages vous aimerez le mieux à la longue." 9

La comparaison entre l'œuvre de l'auteur masculin et celle de Mme de Lambert a pour but de faire briller les qualités de l'œuvre féminine. La solidarité féminine fonctionne bien, les femmes auteurs se renvoient les compliments et références élogieuses : Les Conversations d'Emilie font partie des premières lectures d'Adèle. Mme de Genlis lui donne aussi à lire les Dialogues de Mme de La Fite, Les Lettres péruviennes de Mme Riccoboni en italien, les poésies de Mme Deshoulière, elle évoque le théâtre de Mlle Barbier, et bien sûr les Lettres de Mme de Sévigné. Les femmes auteurs ne sont pas les seules citées; Mme de Genlis ne tarit pas d'éloge par exemple pour Fénelon et son Télémaque et elle loue les romans vertueux de Richardson. Toutefois, elles placent dans leurs programmes de lecture des œuvres de femmes auteurs, sans faire d'écart avec celles des auteurs masculins, et c'est là une évolution notable.

Au sein de ces œuvres, les programmes éducatifs très larges pour des jeunes filles tout comme les références aux œuvres des auteurs féminins célèbres tendent donc à gommer les différences entre homme et femme, à montrer le chemin vers l'égalité des sexes.

\section{Des parcours de femmes éclairées oeuvrant pour la cause des femmes}

Par leurs discours au sein de leurs œuvres que ces femmes auteurs ont contribué à l'avancée des femmes dans la sphère du savoir, mais également par leur parcours dans la shère littéraire Leur acte d'écriture en lui-même a constitué une progression importante pour les femmes dans la société du XVIIIle siècle.

Louise d'Epinay, connue pour ses relations avec Rousseau, Duclos, Diderot, Voltaire et surtout Grimm, a élevé ses enfants avec une attention inaccoutumée dans sa classe sociale. Elle a envoyé ses premiers écrits à Rousseau, Lettres à mon fils, il en a critiqué le style peu

\footnotetext{
${ }^{8}$ Mme d'Epinay, Les Conversations d'Emilie, Quatorzième Conversation, p. 286. Le Théâtre à l'usage des jeunes personnes de Mme de Genlis était paru chez Panckouke en 1779 et chez Lambert et Baudoin en 1780.

${ }^{9}$ Mme d'Epinay, Les Conversations d'Emilie, Seizième conversation, p. 338-339.
} 
adapté à l'enfance. Elle a poursuivi sa tâche d'éducatrice en élevant sa petite fille, Emilie, et en écrivant Les Conversations d'Emilie, centré désormais sur l'éducation féminine. Elle se présente comme une simple femme, une mère ayant élevé plusieurs enfants, qui fait part de cette expérience au public. Cette démarche modeste a plu ; elle a reçu la première le prix Monthyon récompensant l'ouvrage de l'année "dont il pourrait résulter le plus grand bien pour la société" 10 et a été couronnée par l'Académie française en 1783. Elle a eu la primeur sur un livre de Daubenton sur les moutons, un ouvrage de Parmentier sur les pommes de terre et sur Adèle et Théodore de Mme de Genlis ${ }^{11}$.

En lice, face à elle, Caroline de Genlis a connu d'autres honneurs : mère elle-même de deux filles, elle a écrit pour elles des pièces de théâtre qui ont eu du succès dans la bonne société. Elle a pris en charge les deux jumelles de la duchesse de Chartres à l'âge de deux ans et s'est retirée en pleine gloire mondaine du Palais Royal pour les élever à l'écart dans un pavillon spécialement conçu pour ses desseins pédagogiques. En 1781, elle a publié sous son nom Les Annales de la vertu ou cours d'histoire à l'usage des jeunes gens. En 1782, les mille deux cents pages d'Adèle et Théodore font connaître ses théories pédagogiques au moment même où la haute société apprend avec stupéfaction que le duc de Chartres lui confie également l'éducation de ses deux garçons et que pour la première fois une femme est nommée "gouverneur" d'un prince et assure son éducation jusqu'à l'âge adulte. Au pavillon de Bellechasse, elle a donc dirigé une école mixte et formé un futur roi : Louis Philippe. Auteur de 84 livres entre 1779 et 1787, elle a connu un succès plus important encore sous le Consulat et l'Empire. Forte de cette position d'auteur à succès, elle a publié un essai en $1811: D e$ l'influence des femmes dans la littérature ${ }^{12}$, et elle a entrepris -rien de moins- que de réécrire l'Encyclopédie en supprimant toutes les références contraires à la Religion.

Ces femmes sont allées moins loin dans leurs œuvres que dans leur vie... Issues de milieux nobles et aisés, Mmes de Genlis et d'Epinay ont avancé doucement vers le statut de femme écrivain en s'abritant derrière l'utilité de leur tâche d'éducatrice. Dans la fiction de Mme de Genlis, Mme D'Almane, la mère d'Adèle et Théodore, s'excuse de produire toujours de nouveaux romans d'éducation; elle explique que c'est pour suppléer aux manques criants d'ouvrages dans ce domaine, pour le bonheur de ses enfants et pour aider les autres parents. De plus, elle réserve l'éducation de son fils à son mari même si elle écrit aussi pour son fils. Tout le monde n'est pas près à l'époque à accepter une femme dirigeant l'éducation des hommes, la cabale qui a suivi l'annonce de sa nomination au poste de gouverneur l'a bien montré. Des libelles calomniant Mme de Genlis ont circulé en 1782 : ils soupçonnaient la Harpe, d'être l'homme qui écrivait ses textes et le surnommaient "sous-gouvernante" tandis que le duc de Luynes, qui était très gros, était nommé nourrice du dauphin. Bien des femmes, l'ont critiquée pour avoir pris cette fonction traditionnellement masculine. Cependant, Mme de Genlis a continué son œuvre et ses fonctions en insistant dans tous ses discours sur l'importance d'être utile à la société par ses écrits et ses actes.

\footnotetext{
${ }^{10}$ Prix fondé en 1782 par M. de Monthyon pour récompenser "l'ouvrage en prose paru dans l'année, le plus utile et le mieux écrit". Mme d'Epinay présentait le second volume des Conversations d'Emilie, le premier ayant connu un grand succès sept ans plus tôt. Elle était soutenue par tous ses amis encyclopédistes.

${ }^{11}$ Mme de Genlis croyait avoir le prix . Elle avait mené campagne auprès de ses partisans à l'Académie et dénigré l'ouvrage de sa rivale : "un ouvrage plein de fautes de langage, sans intérêt... qui n'est connu que parce que je l'ai loué... qui contient vingt pages de phrases qui ne sont pas françaises et de mots dont les seules femmes de chambre servent. Cependant opposée aux Encyclopédistes par ses conceptions religieuses, elle eut trois fois moins de voix que sa rivale. Voir à ce sujet Gabriel de Broglie, Madame de Genlis (Paris, Librairie Académique Perrin, 1985, p. 117 et suivantes).

${ }^{12}$ De l'influence des femmes sur la littérature française comme protectrices des lettres et comme auteurs ; ou précis de l'histoire des femmes françaises les plus célèbres. Par Mme de Genlis (A Paris, chez Maradan, Libraire, rue des grands-Augustins, $\left.\mathrm{n}^{\circ} 9,1811\right)$.
} 
On peut donc bien parler ici d'écrits qui ont fait évoluer la condition féminine de leur temps. Mme d'Epinay et Mme de Genlis ont innové en présentant des héroïnes, vives, très instruites et néanmoins sages. Elles ont montré dans leurs œuvres que bien éduquées et suivies, des jeunes filles pouvaient suivre sans danger une éducation équivalant à celle des garçons. Leurs mères, fidèles éducatrices à leurs côtés sont aussi porteuses d'une image nouvelle de la femme et notamment de la femme auteur de textes éducatifs, réalisant une œuvre utile pour la société. Ces livres ont servi le parcours personnel de leurs auteurs et leur accès à des honneurs rares pour les femmes de l'époque. Malgré la prégnance des théories de Rousseau en cette fin de siècle, Mme de Genlis et Mme d'Epinay ont donc su se faufiler dans la seule voie légitime pour une femme : l'éducation des enfants et partir de préceptes rousseauistes pour développer des programmes d'éducation féminine ouverts et éclairés, donnant des clés concrètes à leurs lectrices pour faire évoluer la condition des femmes dans la société... ce que n'ont pas fait les hommes des lumières.

Sandrine Aragon -Université de Lille III (lors de l'écritre)

Sorbonne Université CELLF philomèle 